\title{
Optical touch screen based on waveguide sensing
}

Pedersen, Henrik Chresten; Jakobsen, Michael Linde; Hanson, Steen Grüner; Mosgaard, Morten; Iversen, Theis; Korsgaard, Jorgen

Published in:

Applied Physics Letters

Link to article, DOI:

$10.1063 / 1.3615656$

Publication date:

2011

Document Version

Publisher's PDF, also known as Version of record

Link back to DTU Orbit

Citation (APA):

Pedersen, H. C., Jakobsen, M. L., Hanson, S. G., Mosgaard, M., Iversen, T., \& Korsgaard, J. (2011). Optical touch screen based on waveguide sensing. Applied Physics Letters, 99(6), 061102.

https://doi.org/10.1063/1.3615656

\section{General rights}

Copyright and moral rights for the publications made accessible in the public portal are retained by the authors and/or other copyright owners and it is a condition of accessing publications that users recognise and abide by the legal requirements associated with these rights.

- Users may download and print one copy of any publication from the public portal for the purpose of private study or research.

- You may not further distribute the material or use it for any profit-making activity or commercial gain

- You may freely distribute the URL identifying the publication in the public portal

If you believe that this document breaches copyright please contact us providing details, and we will remove access to the work immediately and investigate your claim 


\title{
Optical touch screen based on waveguide sensing
}

\author{
Henrik C. Pedersen, ${ }^{1, a)}$ Michael L. Jakobsen, ${ }^{1}$ Steen G. Hanson, ${ }^{1}$ Morten Mosgaard, ${ }^{2}$ \\ Theis Iversen, ${ }^{2}$ and Jorgen Korsgaard ${ }^{2}$ \\ ${ }^{1}$ DTU Fotonik, Technical University of Denmark, 4000 Roskilde, Denmark \\ ${ }^{2}$ OPDI Technologies A/S, Frederiksborgvej 399, 4000 Roskilde, Denmark
}

(Received 4 April 2011; accepted 1 July 2011; published online 9 August 2011)

\begin{abstract}
We disclose a simple, optical touch screen technique based on a planar injection molded polymer waveguide, a single laser, and a small linear detector array. The solution significantly reduces the complexity and cost as compared to existing optical touch technologies. Force detection of a touching finger is also demonstrated. (C) 2011 American Institute of Physics. [doi:10.1063/1.3615656]
\end{abstract}

The introduction of Apple's iPhone in 2007 was indeed responsible for a breathtaking development of touch screens. Not only did the worldwide production of touch screens explode ( $>400 \mathrm{M}$ units in 2009 (Ref. 1)) but the technical evolution experienced a great boost as well. Features like dual touch (allowing magnifying/rotating pictures), internet and e-mail access, and advanced gaming were all contributing to raise the bar for the touch screens to come. On top of that there has been an on-going development towards larger screens, such as tablet computers and laptops.

Today, at least a dozen different touch screen technologies have made it to the market; however, two technologies are dominating: resistive and projected capacitive. ${ }^{1}$ Resistive is still the preferred technology due to low price, but projected capacitive is catching up especially for portable devices like smart phones.

Optical touch screens have been available on the market for more than $40 \mathrm{yr}^{2}{ }^{2}$ The classical types, known from, e.g., industrial control panels, rely on an "over-the-surface" light grid, in which two arrays of light emitters arranged along two adjacent sides of the display pass light above the display towards corresponding arrays of light detectors at the opposite sides. When a finger touches the display, two orthogonal light beams are blocked, respectively, in the $\mathrm{x}$ and $\mathrm{y}$ directions, leading to an immediate determination of the finger location. Such systems do have certain advantages over capacitive touch screens used in many handheld devices today (e.g., Apple's iPhone), in that no force needs to be exerted on the screen and no electrically conducting coatings need to be applied to the screen, the latter reducing the visibility of the display. Moreover, the optical solution is easily scalable to large size, unlike the electronic solutions. There are, however, also several draw-backs of the over-the-surface solutions, such as sensitivity to ambient light, high cost, and complexity (because of the large amount of light sources and detectors) and the fact that a bezel is needed around the edge of the display, which especially for handheld devices is undesirable.

There is also a second type of optical touch screen in which the light beams are guided in a planar waveguide placed right on top of the display, ${ }^{3-5}$ see Fig. 1 . The light blocking then relies upon locally disturbing the total-inter-

\footnotetext{
${ }^{\text {a) }}$ Author to whom correspondence should be addressed. Electronic mail: hcpe@fotonik.dtu.dk.
}

nal-reflection, much in the same way as in refractive-index waveguide sensing. ${ }^{6}$ The angle of interrogation $\theta$ is chosen between

$$
\theta_{c, i l}<\theta<\theta_{c, f}, \theta_{c, i l}=\arcsin \left(\frac{n_{i l}}{n_{w g}}\right), \theta_{c, f}=\arcsin \left(\frac{n_{f}}{n_{w g}}\right),
$$

where $\theta_{c, i l}$ is the critical angle at the isolation layer separating the waveguide from the display, $\theta_{c, f}$ is the critical angle at the finger, and $n_{i l, w g . f}$ are the refractive indices of isolation layer, waveguide, and finger, respectively. If Eq. (1) is fulfilled, light may be picked up by a touching finger, which leaves the edge-detector dark. Hence, by arranging arrays of light emitters along two sides of the waveguide and arrays of detectors along the two opposite edges, the touch location is easily derived from the two dark detectors. However, due to the large amount of light sources and detectors needed to cover the entire display area, this type of touch screen was never commercialized.

The objective of the present paper is to present a waveguide-based optical touch screen in which the amount of light sources and light detectors is significantly reduced.

The touch screen is based on the same total-internalreflection principle, as shown in Fig. 1. However, we use only a single light source and a small detector array, as shown in a top view in Fig. 2. The touch module comprises a transparent plastic plate (PMMA), acting as the waveguide, a single laser $V$ (VCSEL, vertical cavity surface emitting laser) placed at one corner of the waveguide and a small linear detector array D.A. placed at the opposite corner. The laser light is coupled into the waveguide through a concave, cylindrical lens-shape molded into the corner. The cylindrical lens expands the laser beam into a $\pm 45^{\circ}$ fan, so that the entire plane of the waveguide is illuminated. The light propagates in the waveguide towards the two opposite collimating edges $C_{x, y}$ that are shaped as Fresnel-parabolas and are

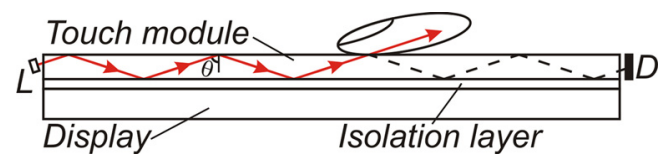

FIG. 1. (Color online) Principle of a waveguide-based touch screen. $L=$ light source, $D=$ detector, $\theta=$ angle of interrogation. 


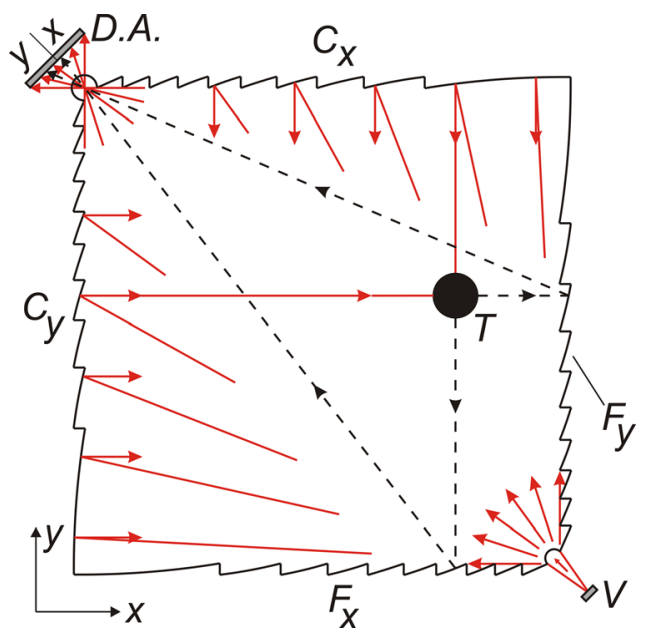

FIG. 2. (Color online) Top view of the touch module comprising a polymer waveguide with specially shaped edges, a light source $V$, and a linear detector array D.A. $C_{x, y}$ are Fresnel-shaped collimating edges, $F_{x, y}$ are Fresnelshaped focusing edges, and $T$ is a touch point. The two $x$ - and $y$-parts of the detector array are dedicated to the $x$ - and $y$-interrogating rays, respectively.

coated with gold. Here, the beams are redirected and collimated along the $-y$ and $+x$ axes, respectively, see Fig. 2. At the opposite edges $F_{x, y}$, the beams are redirected and focused at the out-coupling corner (upper left), at which a convex, cylindrical shape carries out the out-coupling towards the $x$ and $y$-parts of the detector array.

Upon touching the surface at position $T$, the total internal reflection is disturbed in the same manner as illustrated in Fig. 1. As a result, two dark spots are generated at the detector array, which readily gives the $x$ and $y$ coordinates of the touch. This waveguide design solves the problems encountered by the current optical touch screens, as the amount of light sources and detectors are reduced significantly and there is no bezel needed along the edges.

There is, however, a fundamental issue to be taken care of, since not only the collimated rays are sensitive to touch. This is illustrated further in Fig. 3 showing that also the diverging rays $D$ are disturbed by the touch. This leads to

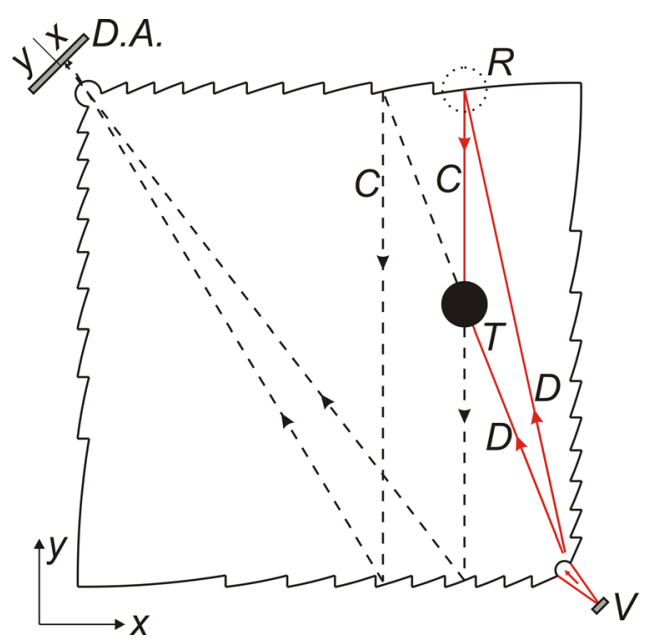

FIG. 3. (Color online) The fact that both the collimated rays $C$ and the diverging rays $D$ are touch sensitive leads to unintended, multiple dark spots at the detector array, here illustrated by the two dark spots appearing on the $\mathrm{x}$-part of the detector array. Similarly, two dark spots will appear on the y-part.

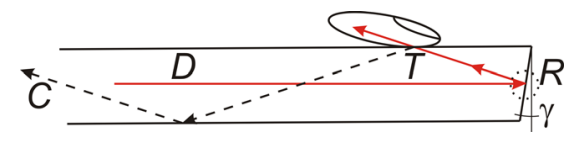

FIG. 4. (Color online) Cross-sectional view at the reflection point $R$ in Fig. 3. The tilted edge at $R$ alters the angle of interrogation of the reflected ray, making it touch sensitive.

two dark " $x$-spots" at the detector array instead of one. In addition, the focusing rays are also touch sensitive, giving rise to two dark " $y$-spots." Hence, a single touch leads to a total of four dark spots at the detector array, making the detector information ambiguous.

Our way of solving this problem is to introduce a tilt to the Fresnel-shaped edges, which is shown in Fig. 4. Here, the diverging ray $D$ is incident virtually along the plane of the waveguide, meaning that the angle of interrogation is close to $90^{\circ}$. The critical angle for the PMMA/finger interface is $\arcsin (1.47 / 1.49)=80.6^{\circ},{ }^{7}$ meaning-according to Eq. (1) - that the diverging rays are not touch sensitive. After being reflected, the interrogation angle is decreased by $2 \gamma$, where $\gamma$ is the tilt angle of the edge. In our case, the edge is tilted by $9^{\circ}$, meaning that the interrogation angles of the collimated rays are around $72^{\circ}$, i.e., well below the required $80.6^{\circ}$. Thus, the collimated rays become touch sensitive, as illustrated in Fig. 4.

The focusing edges are tilted oppositely, so that the interrogation angles of the focusing rays are also close to $90^{\circ}$, making them non-sensitive to touch. In this manner, the unintended dark spots are eliminated, leading to the desired two-spot, unambiguous detector signal.

The realized PMMA waveguide is photographed in Fig. 5. The dimensions are $40 \times 40 \times 1 \mathrm{~mm}$; the Fresnel structures are $0.5 \mathrm{~mm}$ deep. As an illustration, the transition from a nonsensitive, in-the-plane ray to a sensitive zig-zag ray is shown. The single zig-zag ray shown may leave the impression that there are only distinct points of interrogation at the surface. However, in reality, a fan of rays with an angular range of $\sim \pm 4^{\circ}$ are launched into the waveguide. This smears out the interrogation points to form a continuum. The waveguide is therefore touch-sensitive all over the surface.

To test the touch performance, the waveguide was mounted on a block of polytetrafluorethylen, which has a refractive index of 1.35 and therefore is well suited as an

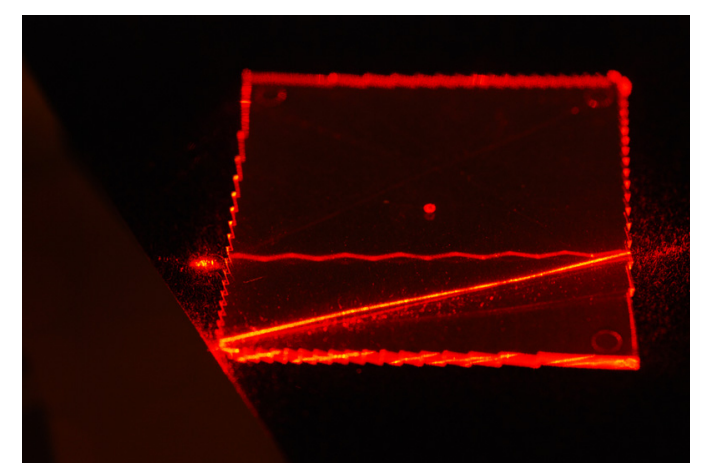

FIG. 5. (Color online) Photograph of the PMMA waveguide illustrating the effect of the tilted Fresnel-shaped edge: an in-the-plane ray originating from the lower left corner is transformed into a zig-zag ray with interrogation angle $=72^{\circ}$. 

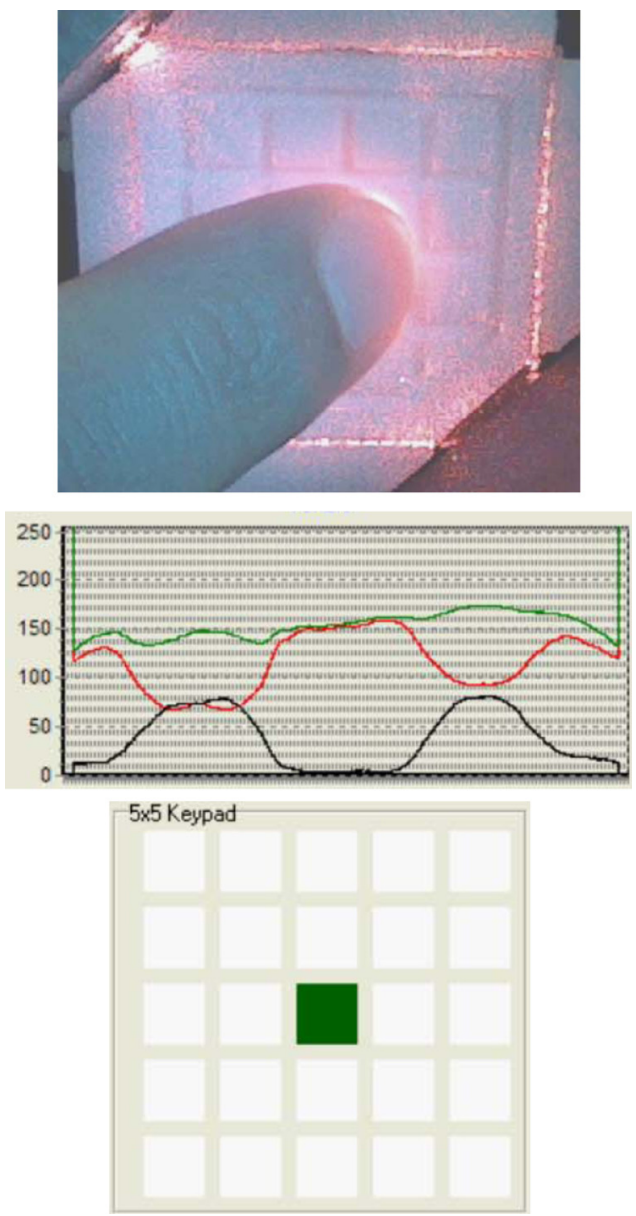

FIG. 6. (Color online) (a) Realized touch module being tested. Laser light is being launched at the upper left corner; the camera is placed at the opposite corner. Note the "glow" at the tip of the finger, which is light being picked up from the waveguide. (b) Green curve (top curve) is the un-touched camera signal, red curve (middle curve) is the touched camera signal, black curve (bottom curve) is a result of a subtraction between the green and red curves. (c) Result of the peak finding algorithm that tells the touch location.

isolating substrate. A VCSEL (Optowell SS67-4U001, single mode, $\lambda=670 \mathrm{~nm}$ ) was used as a light source and a complementary metal-oxide-semiconductor (CMOS) camera (EHD, SMX-160, 6.6 Mpixels) was used as a detector array. The in-coupling lens-shape had a radius of $0.2 \mathrm{~mm}$, and the outcoupling lens-shape had a radius of $3 \mathrm{~mm}$.

The touch module was tested by touching with a finger at different locations, as shown in Fig. 6(a). By subtracting the resulting detector signal from the reference frame (=signal without touch), two peaks appear, as shown in Fig. 6(b). An algorithm for peak finding was developed to locate the peaks and from this calculate the finger location, which is shown in Fig. 6(c). Based on this, a $5 \times 5$ keypad was realized and tested. Furthermore, sliding movements across the device were tracked by the algorithm.

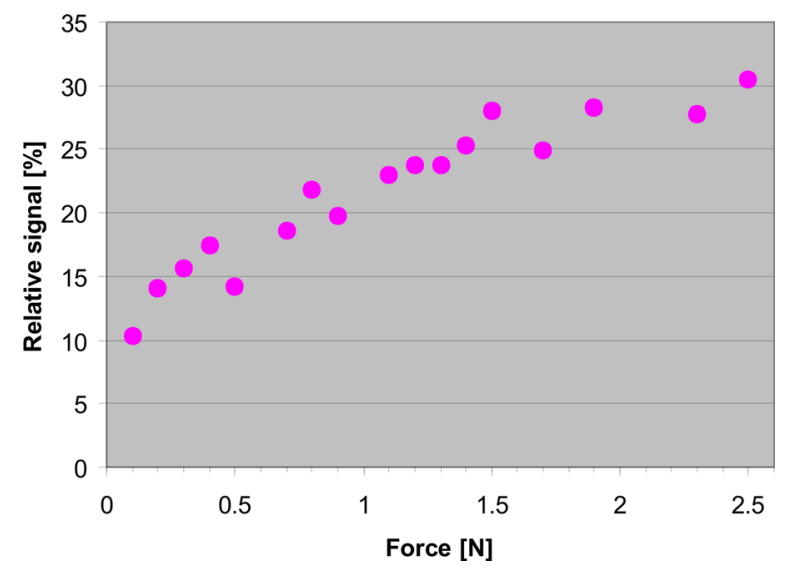

FIG. 7. (Color online) Average, relative peak-height changes (in relation to reference signal) of the $x$ and $y$ peaks versus force applied by the finger.

The influence of the touch force was also investigated. The device was placed on a scale and the signals resulting from different touch forces were recorded. The results are shown in Fig. 7, revealing that a threshold force of $0.1 \mathrm{~N}$ (10 grams) was needed to obtain a $10 \%$ local change of the signal. The relative signal increases almost linearly up to $2.5 \mathrm{~N}$. The force sensitivity is very interesting for future touch screens, in which three-dimensional inputs $(x, y, z)$ could be offered as a feature. One example is navigating a mouse in three dimensions or controlling an object in $3 \mathrm{D}$ space. $^{8}$

In conclusion, we have reported an optical touch technology that considerably reduces the cost compared to existing optical technologies. Force detection is also demonstrated, a feature that may add a third dimension to the existing smart phone interactions. We believe that the presented technology is attractive for the high-volume touch screen market.

This work was supported by the Danish Council for Technology and Innovation under the Innovation Consortium CINO (Centre for Industrial Nano Optics), Grant No. 75823.

${ }^{1}$ M. J. Miller, PCMAG, available at http://www.pcmag.com/article2/ 0,2817,2360930,00.asp (March 3 2010).

${ }^{2}$ I. Maxwell, Inf. Disp. 12, 26 (2007).

${ }^{3}$ R. G. Johnson and D. Fryberger, U.S. patent 3,673,327 (1972).

${ }^{4}$ H. Ma and J. A. Paradiso, U.S. patent 2004/0252091 (2004).

${ }^{5} \mathrm{~J}$. Moeller and A. Kerne, Proceedings of the Fourth International Conference on Tangible, Embedded, and Embodied Interaction, January 25-27 (ACM, Cambridge, Massachusetts, 2010), p. 73.

${ }^{6}$ R. Horvath, N. Skivesen, and H. C. Pedersen, Appl. Phys. Lett. 84, 4044 (2004).

${ }^{7}$ The refractive index of a finger was found in A. Knüttel and M. BoehlauGodau, J. Biomed. Opt. 5, 83 (2000).

${ }^{8}$ D. A. Soss, Inf. Disp. 12, 20 (2007). 